\title{
AN APPLICATION OF ASYMPTOTIC TECHNIQUES TO CERTAIN PROBLEMS OF SPECTRAL AND SCATTERING THEORY OF STARK-LIKE HAMILTONIANS
}

\author{
BY
}

\author{
MATANIA BEN-ARTZI
}

\begin{abstract}
Let $L_{0}=-\Delta+V\left(x_{1}\right), L=L_{0}+V_{p}(x)$ be selfadjoint in $L^{2}\left(R^{n}\right)$. Here $V, V_{p}$ are real functions, $V\left(x_{1}\right)$ depends only on the first coordinate. Existence of the wave-operators $W_{ \pm}\left(L, L_{0}\right)=s$ - $\lim _{t \rightarrow \pm \infty} \exp (i t L) \exp \left(-i t L_{0}\right)$ is proved, using the stationary phase method. For this, an asymptotic technique is applied to the study of $-d^{2} / d t^{2}+V(t)$ in $L^{2}(R)$. Its absolute continuity is proved as well as a suitable eigenfunction expansion. $V$ is a "Stark-like" potential. In particular, the cases $V\left(x_{1}\right)=\left(-\operatorname{sgn} x_{1}\right)\left|x_{1}\right|^{\alpha}, 0<\alpha \leqslant 2$, are included. $V_{p}$ may be taken as the sum of an $L^{2}$-function and a function satisfying growth conditions in the $+x_{1}$ direction. $V_{p}(x)=|x|^{-1}$ is included.
\end{abstract}

I. Introduction. It is the purpose of this paper to present an application of a new asymptotic technique to the study of certain problems which are related to the Stark Hamiltonian $-\Delta-x_{1}$.

Consider first a one-dimensional Schrödinger operator $H_{0}=-d^{2} / d t^{2}+V(t)$ in $L^{2}(R)$, where $V(t)$ is a real function. $H_{0}$ is symmetric when defined on $C_{0}^{\infty}(R)$. An extensive study of its selfadjoint extensions is given in [7]. Rejto and Sinha [14] used the JWKB method to prove the absolute continuity of $H_{0}$, subject to certain assumptions on the relative decay of $V^{\prime}, V^{\prime \prime}$ with respect to $V$. Our model is closely related to [14]. We allow the leading term in $V$ to behave as $\pm|x|^{2}$ as $x \rightarrow \mp \infty$. Roughly speaking, we assume that this term is smooth and that the relative rate of decay of its derivatives at infinity improves with successive differentiation. The detailed assumptions on $V$ are listed in $\S I I$.

In §III we state three asymptotic lemmas which constitute the main technical tool in our study. They are concerned with questions of existence and regularity of asymptotic (oscillatory or exponentially decaying) solutions of the equation $H_{0} y=z y$ where $z$ is in a neighborhood of the spectrum. Precise asymptotic expansions are obtained, which in a certain sense are generalizations of the Jost solutions [13, $\S X I .8]$. The proofs of the lemmas are sketched in the Appendix.

In §IV we study the operator $L_{0}=-\Delta+V\left(x_{1}\right)$ in $L^{2}\left(R^{n}\right)$, where $V\left(x_{1}\right)$ is as above. In fact, we note that by standard function-analytic theorems we only have to deal with $H_{0}$ in $L^{2}(R)$. Utilizing the asymptotic lemmas we prove the essential

Received by the editors August 27, 1982.

1980 Mathematics Subject Classification. Primary 35P25, 81F05.

(C) 1983 American Mathematical Society 0002-9947/82/0000-1083/\$05.75 
selfadjointness of $L_{0}$. Also assuming that

$$
\lim _{t \rightarrow+\infty} V(t)=-\infty, \quad \lim _{t \rightarrow-\infty} \inf V(t)=\Gamma>-\infty
$$

we prove the absolute continuity of $H_{0}$ over $(-\infty, \Gamma)$. We go beyond that and establish an eigenfunction expansion theorem for the part of $H_{0}$ in $(-\infty, \Gamma)$. Thus, for example, we show that the opertors $-d^{2} / d t^{2}-(\operatorname{sgn} t)|t|^{\alpha}, 0<\alpha \leqslant 2$, are all absolutely continuous (and, in fact, unitarily equivalent) over $(-\infty, \infty)$. A Coulomblike perturbation can be added to each of them without changing this result.

In $\S \mathrm{V}$ we add a multidimensional perturbation $V_{p}(x)$ and study the existence of the associated (partial) wave operators for $L_{0}, L=L_{0}+V_{p}$, defined by,

$$
W_{ \pm}=\operatorname{sim}_{t \rightarrow \pm \infty} \exp (i t L) \exp \left(-i t L_{0}\right) E_{0}(-\infty, \Gamma)
$$

where $\left\{E_{0}(\lambda)\right\}$ is the spectral family of $L_{0}$. We combine here Cook's method and stationary phase estimates [10] for the eigenfunction expansions. Note that in the Stark case, $V\left(x_{1}\right)=-x_{1}$, the generalized eigenfunctions are Airy functions.

$\S \mathrm{VI}$ is a specialization of the theorems of the previous sections to the family of operators having the form

$$
T_{\alpha}=-\Delta-\left(\operatorname{sgn} x_{1}\right)\left|x_{1}\right|^{\alpha}, \quad 0<\alpha \leqslant 2 .
$$

As stated earlier, they are all absolutely continuous and unitarily equivalent. However, due to differences in some features of their eigenfunction expansions, the assumptions imposed on a multidimensional perturbation $V_{p}(x)$ in order to ensure the existence of the wave-operators depend on $\alpha$. For the Stark Hamiltonian, $\alpha=1$, in $L^{2}\left(R^{3}\right)$ we obtain the following

Theorem. Let $T_{1}=-\Delta-x_{1}+V_{S}\left(x_{1}\right)$, where $V_{S}\left(x_{1}\right) \in L^{2}(R)$ and decays as $\left|x_{1}\right|^{-3 / 2-\varepsilon}$ as $\left|x_{1}\right| \rightarrow \infty$. Let $V_{p}(x)$ be a real potential such that for some $N$, $(1+|x|)^{-N} V_{p}(x) \in L^{2}\left(R^{3}\right)$ and such that $V_{p}=V_{1}+V_{2}$ where

(a) $V_{1}(x) \in L^{2}\left(R^{3}\right)$

(b) $\left|V_{2}\left(x_{1}, x^{\prime}\right)\right| \leqslant C\left(1+\left|x^{\prime}\right|\right)^{r}\left(1+x_{1}\right)^{-s}, x_{1} \geqslant 0$, where $x=\left(x_{1}, x^{\prime}\right), r \geqslant 0$ and $s>\frac{1}{2}(r+1)$.

Let $T$ be any selfadjoint extension of $\left(T_{1}+V_{p}\right) / C_{0}^{\infty}\left(R^{n}\right)$. Then the wave-operators $W_{ \pm}\left(T, T_{1}\right)$ exist.

This result was first obtained by Avron and Herbst [1]. The existence of the wave-operators for the Stark case was also proved by Veselic and Weidmann [17]. The problem of completeness of the wave operators (i.e., that Range $W_{ \pm}=$subspace of absolute continuity with respect to $L$ ) is of course more delicate. Since in this case the absolutely continuous parts of $L, L_{0}$ are unitarily equivalent one can expect some further "smallness" assumptions on the perturbation potential $V_{p}$ with respect to $V$. This has been carried out only for the Stark case by Herbst [9], Simon [16] and Yajima [18]. Their methods, although considerably different from each other, all make use of some explicit properties of the Stark potential, such as the expansion by Airy functions and an explicit unitary transformation eliminating $-d^{2} / d x_{1}^{2}$. In a 
separate paper [3] we use our asymptotic methods in order to derive a limiting absorption principle and establish the completeness of the wave-operators for a more general "Stark-like" Hamiltonian.

II. Assumptions and notations. Let $k$ be a positive integer, $\delta>0$. We denote by $B(k, \delta ;+\infty)$ the set of functions $f(x)$ defined in a neighborhood of $+\infty$ (which may depend on $f$ ) and such that

$$
\begin{aligned}
f(x) & \in C^{2 k}\left(x_{0}, \infty\right), \\
(1+|f(x)|)^{-1}\left(\frac{d}{d x}\right)^{j} f(x) & =O\left(x^{-j \delta}\right) \text { as } x \rightarrow+\infty, 1 \leqslant j \leqslant 2 k .
\end{aligned}
$$

We shall always assume that $2 k \delta>1$. The set $B(k, \delta ;-\infty)$ is defined similarly. Let

$$
L_{0}=-\Delta+V\left(x_{1}\right), \quad L=-\Delta+V\left(x_{1}\right)+V_{p}(x), \quad x=\left(x_{1}, x^{\prime}\right) \in R^{n},
$$

be Schrödinger operators in $R^{n}$, where the potential $V\left(x_{1}\right)$ depends on $x_{1}$ only. Our assumptions on $V\left(x_{1}\right)$ are listed below in detail, whereas the exact assumptions on $V_{p}(x)$, the "perturbation" potential, will be given later (see $\S \mathrm{V}$ ).

$$
V(t) \in L_{\mathrm{loc}}^{2}(R) \quad(R \equiv(-\infty, \infty)),
$$

and can be decomposed as

$$
V(t)=V_{L}(t)+V_{S}(t),
$$

(to which we shall refer as the "long-range" and "short-range" parts) where

$$
\begin{aligned}
& \text { (VL1) } V_{L}(t) \in B(k, \delta ; \pm \infty) \text { for suitable } k, \delta \text { with } 2 k \delta>1 . \\
& \text { (VL2) } \lim _{t \rightarrow+\infty} V_{L}(t)=-\infty<\liminf _{t \rightarrow-\infty} V_{L}(t)=\Gamma \quad(\text { we allow } \Gamma=+\infty) . \\
& \text { (VL3) } \int_{1}^{\infty} \frac{d t}{1+\left|V_{L}(t)\right|^{1 / 2}}=\infty, \quad \int_{-\infty}^{-1} \frac{d t}{1+\left|V_{L}(t)\right|^{1 / 2}}=\infty
\end{aligned}
$$

(VS) $V_{S}(t)$ is short-range with respect to $V_{L}(t)$, i.e., for some $\varepsilon>0$

$$
\left|V_{S}(t)\right| \times\left(1+\left|V_{L}(t)\right|\right)^{-1 / 2}=O\left(|t|^{-1-\varepsilon}\right) \quad \text { as }|t| \rightarrow \infty .
$$

We do not make any attempt to get the best possible estimates for $V_{S}(t)$, since it will be used primarily to allow for finite singularities.

Observe that our assumptions allow for potentials of the type

$$
V_{L}(t)=-(\operatorname{sgn} t) \cdot|t|^{\alpha}, \quad 0<\alpha \leqslant 2,
$$

and, in particular, the Stark potential $(\alpha=1)$ is included.

We shall study the behavior of the operators $L, L_{0}$ over the interval

$$
\Lambda=(-\infty, \Gamma) \text {. }
$$

For an interval $I=[\alpha, \beta] \subseteq \Lambda$ and a small $\eta>0$ we use the notation

$$
\Omega(I)=\{z \mid \alpha \leqslant \operatorname{Re} z \leqslant \beta, 0 \leqslant \operatorname{Im} z \leqslant \eta\} .
$$


III. Some asymptotic lemmas. Our main tool consists of the following asymptotic lemmas, which characterize the behavior of one-dimensional solutions of the equation:

$$
\left(-\frac{d^{2}}{d t^{2}}+V(t)\right) y(t)=z y(t), \quad z \in \Omega(I), I \subseteq \Lambda .
$$

We shall find it convenient to denote by $\delta^{ \pm}(t, z)$ a (generic) function continuous on $\left[t_{0}, \infty\right) \times \Omega(I)\left(\left(-\infty, t_{0}\right] \times \Omega(I)\right)$ for $t_{0}$ sufficiently large, and such that

$$
\begin{aligned}
\left|\delta^{ \pm}(t, z)\right| \leqslant C\left(\left|\int_{t}^{ \pm \infty}\right| V_{S}(\xi) \mid\right. & \left(1+\left|V_{L}(\xi)\right|^{1 / 2}\right)^{-1} d \xi \mid \\
& \left.+\left|\int_{t}^{ \pm \infty}\left(1+\left|V_{L}(\xi)\right|\right)^{-k+1 / 2}(1+|\xi|)^{-2 k \delta} d \xi\right|\right),
\end{aligned}
$$

for a suitable constant $C$ (depending on $I, \delta$ ).

LEMMA 3.1. Equations (3.1) has a solution $y^{+}(t, z)$, which is continuous on $R \times \Omega(I)$ and satisfies, for $z \in \Omega(I)$

$$
\begin{aligned}
y^{+}(t, z)= & \left(z-V_{L}(t)\right)^{-1 / 4}(1+a(t, z))^{-1 / 2} \\
& \cdot\left\{\exp \left(i \int_{t_{0}}^{t}(1+a(s, z))\left(z-V_{L}(s)\right)^{1 / 2} d s\right) \cdot\left(1+\delta^{+}(t, z)\right)\right\} . \\
\frac{d y^{+}}{d t}(t, z)= & i\left(z-V_{L}(t)\right)^{1 / 4}(1+a(t, z))^{1 / 2} \\
& \cdot\left\{\exp \left(i \int_{t_{0}}^{t}(1+a(s, z))\left(z-V_{L}(s)\right)^{1 / 2} d s\right) \cdot\left(1+\delta^{+}(t, z)\right)\right\} \\
& +y^{+}(t, z) \cdot\left\{\frac{1}{4} V_{L}^{\prime}(t)\left(z-V_{L}(t)\right)^{-1}-\frac{1}{2}(1+a)^{-1} \frac{d a}{d t}\right\},
\end{aligned}
$$

as $t \rightarrow+\infty$, where $a(t, z)$ has the following properties;

(a) $a(t, z)$ depends on $V_{L}$ only (i.e., not on $\left.V_{S}\right)$ and is continuous on $\left[t_{0}, \infty\right) \times \Omega(I)$.

(b) $\operatorname{Im} a(t, z)=0$ if $\operatorname{Im} z=0$.

(c) $|a(t, z)|+|d a / d t|=O\left(\left|z-V_{L}(t)\right|^{-1} t^{-2 \delta}\right)$ as $t \rightarrow+\infty$, uniformly in $z \in \Omega(I)$.

(d) For every fixed $t, a(t, \lambda)$ is infinitely differentiable with respect to the real variable $\lambda \in I$ and for every positive integer $m$

$$
\left|(d / d \lambda)^{m} a(t, \lambda)\right|=O\left(\left|\lambda-V_{L}(t)\right|^{-(m+1)} t^{-2 \delta}\right),
$$

as $t \rightarrow+\infty$, uniformly in $\lambda \in I$.

REMARK. Note that since $\operatorname{Re} z>\lim _{t \rightarrow+\infty} V_{L}(t)$ we can rearrange $V=V_{L}+V_{S}$ so that for $t \geqslant t_{0}$ we have $\operatorname{Re} z>V_{L}(t)$ and all roots of $z-V_{L}(t)$ are well defined, when the principal branch of the logarithm is taken. 
LEMMA 3.2. Equation (3.1) has a solution $y^{-}(t, z)$ which is continuous on $R \times \Omega(I)$ and satisfies, for $z \in \Omega(I)$

$$
\begin{aligned}
y^{-}(t, z)= & \left(V_{L}(t)-z\right)^{-1 / 4}(1+b(t, z))^{-1 / 2} \\
& \cdot\left\{\exp \left(-\int_{t}^{-t_{0}}\left(V_{L}(s)-z\right)^{1 / 2}(1+b(s, z)) d s\right) \cdot\left(1+\delta^{-}(t, z)\right)\right\} . \\
\frac{d y^{-}}{d t}(t, z)= & \left(V_{L}(t)-z\right)^{1 / 4}(1+b(t, z))^{1 / 2} \\
& \cdot\left\{\exp \left(-\int_{t}^{-t_{0}}\left(V_{L}(s)-z\right)^{1 / 2}(1+b(s, z)) d s\right) \cdot\left(1+\delta^{-}(t, z)\right)\right\} \\
& +y^{-}(t, z) \cdot\left\{\frac{1}{4} V_{L}^{\prime}(t)\left(V_{L}(t)-z\right)^{-1}+\frac{1}{2}(1+b)^{-1} \frac{d b}{d t}\right\},
\end{aligned}
$$

as $t \rightarrow-\infty$, where $b(t, z)$ depends on $V_{L}$ only and has the following properties:

(a) $b(t, z)$ is continuous on $\left(-\infty,-t_{0}\right] \times \Omega(I)$.

(b) $\operatorname{Im} b(t, z)=0$ if $\operatorname{Im} z=0$.

(c) $|b(t, z)|+|d b / d t|=O\left(\left|V_{L}(t)-z\right|^{-1}|t|^{-2 \delta}\right)$ as $t \rightarrow-\infty$, uniformly in $z \in$ $\Omega(I)$.

Here we can assume $\operatorname{Re} z \leqslant V_{L}(t)$ and for the roots we take again the principal branch of the logarithm. In particular, $\operatorname{Re}\left(V_{L}(s)-z\right)^{1 / 2}>0$.

Our next lemma is concerned with the question of the differentiability of the solutions $y^{ \pm}(t, z)$ with respect to $z$. Due to the different behavior at $\pm \infty$ (i.e., oscillatory vs. exponentially decaying), the differentiability of $y^{+}(t, z)$ is more restricted than that of $y^{-}(t, z)$. Define the function

$$
w(t)=\int_{t_{0}}^{t}\left(1+\left|V_{L}(\xi)\right|\right)^{-1 / 2} d \xi, \quad t_{0} \leqslant t<\infty
$$

We have

LEMMA 3.3. (a) For a positive integer $m$ suppose that

$$
\begin{aligned}
q(\xi) \equiv w(\xi)^{m} \cdot\left\{\left|V_{S}(\xi)\right|\right. & \left(1+\left|V_{L}(\xi)\right|^{1 / 2}\right)^{-1} \\
& \left.+\left(1+\left|V_{L}(\xi)\right|\right)^{-k+1 / 2}(1+|\xi|)^{-2 k \delta}\right\} \in L^{1}\left(t_{0}, \infty\right),
\end{aligned}
$$

then for every fixed $t \in\left[t_{0}, \infty\right)$ the functions $y^{+}(t, z), d y^{+}(t, z) / d t$ are m-times continuously differentiable with respect to the real variable $z \in I$.

Furthermore, in this case the asymptotic expansion (3.3) can be differentiated m-times successively, with

$$
\left|\left(\frac{d}{d \lambda}\right)^{j} \delta^{+}(t, \lambda)\right| \leqslant C \int_{t}^{\infty} w(\xi)^{j-m} q(\xi) d \xi,
$$


for $0 \leqslant j \leqslant m, t_{0} \leqslant t<\infty$ and $\lambda \in I$. Otherwise stated,

$$
\begin{aligned}
y^{+}(t, \lambda)= & \left(\lambda-V_{L}(t)\right)^{-1 / 4}(1+a(t, \lambda))^{-1 / 2} \\
& \cdot\left\{\exp \left(i \int_{t_{0}}^{t}\left(\lambda-V_{L}(s)\right)^{1 / 2}(1+a(s, \lambda)) d s\right)+r^{+}(t, \lambda)\right\}
\end{aligned}
$$

where the "perturbation" $r^{+}(t, \lambda)$ is m-times differentiable with respect to $\lambda \in I$ and

$$
\left|\left(\frac{d}{d \lambda}\right)^{j} r^{+}(t, \lambda)\right| \leqslant C \int_{t}^{\infty} w(\xi)^{j-m} q(\xi) d \xi,
$$

for $0 \leqslant j \leqslant m, t_{0} \leqslant t<\infty, \lambda \in I$.

(b) Suppose that for every $\mu>0$

$$
\limsup _{t \rightarrow-\infty}\left|V_{L}(t)\right| e^{\mu t} \leqslant 1,
$$

then there exists a neighborhood $U$ of $I$ in the complex plane such that for every fixed $-\infty<t \leqslant-t_{0}$ the function $y^{-}(t, z)$ can be extended as an analytic function of $z \in U$. Furthermore, there exists a $\beta>0$, depending only on $U$, such that for every positive integer $m$

$$
\left|\left(\frac{d}{d z}\right)^{m} y^{-}(t, z)\right| \leqslant C_{m} e^{-\beta|t|}
$$

for $-\infty<t \leqslant-t_{0}, z \in U \cap \Omega(I)$, where $C_{m}$ is a constant depending only on $m, U$.

Lemma 3.1 is proved in [2, II]. The proof of Lemma 3.2 is quite similar. Both proofs are outlined in the Appendix, where the proof of Lemma 3.3 is given too.

IV. Spectral structure of $L_{0}$. Let $L_{0}$ be the operator in $L^{2}\left(R^{n}\right)$ given by (2.2). Clearly, $L_{0}$ is well defined and symmetric on $C_{0}^{\infty}\left(R^{n}\right)$. As a matter of fact, we have

LEMMA 4.1. $L_{0}$ restricted to $C_{0}^{\infty}\left(R^{n}\right)$ is essentially selfadjoint, i.e., its closure is a selfadjoint operator in $L^{2}\left(R^{n}\right)$.

Proof. Note that

$$
\begin{gathered}
L_{0}=L_{0,1} \otimes I+I \otimes L_{0}^{\prime}, \\
L_{0,1}=-d^{2} / d x_{1}^{2}+V\left(x_{1}\right), \quad L_{0}^{\prime}=-\Delta \text { in } R_{x^{\prime}}^{n-1} .
\end{gathered}
$$

By a standard theorem on tensor products $[12, \S$ VIII.10] it suffices to show that $L_{0,1}$ is essentially selfadjoint on $C_{0}^{\infty}(R)$. By the Weyl-Kodaira Theorem [7, §XIII.2] it is enough to show that it has no boundary values at $\pm \infty$. But from assumption (VL3) and Lemma 3.1 it follows that $y^{+}(t, \lambda)$, for $\lambda \in \Lambda$, is not square integrable near $+\infty$. Also, the same assumption and the remark following the proof of Lemma 3.2 in the Appendix imply that there exists a non-square-integrable solution of equation (3.1) near $-\infty$. The lemma is proved in view of Theorem XIII.6.11 in [7]. Q.E.D.

Note that since no pointwise boundedness of $V_{S}$ has been assumed, we could not use a simpler argument based on the lower boundedness of $V($ near $-\infty)$.

The unique selfadjoint extensions will still be denoted by $L_{0}, L_{0,1}, L_{0}^{\prime}$. 
We now proceed to study the structure of the spectrum of $L_{0}$ in $\Lambda=(-\infty, \Gamma)$. Observe that by (4.1) $L_{0}$ is spectrally absolutely continuous for $n>1$ (the convolution of an absolutely continuous measure and a measure is again absolutely continuous). Therefore we concentrate on $L_{0,1}$.

LEMMA 4.2. $\Lambda$ is contained in $\sigma_{e}\left(L_{0,1}\right)$, the essential spectrum of $L_{0,1}$.

Proof. Let $T_{0}$ be the restriction of $L_{0,1}$ to $[0, \infty)$, together with a boundary condition at 0 such that $T_{0}$ is selfadjoint in $L^{2}(0, \infty)$. By Theorem XIII.7.4 in [7] $\sigma_{e}\left(T_{0}\right) \subseteq \sigma_{e}\left(L_{0,1}\right)$. On the other hand, using the proof of Theorem 3.1 in [2, II] we have $\Lambda \subseteq \sigma_{e}\left(T_{0}\right)$. Q.E.D.

THEOREM 4.1. The spectrum of $L_{0,1}$ over $\Lambda$ is absolutely continuous.

Proof. Let $I \subseteq \Lambda$ be a closed interval. For nonreal $z \in \Omega(I)$ let $R(z)=$ $\left(L_{0,1}-z I\right)^{-1}$ and let $K(t, s ; z)$ be its kernel, namely;

$$
R(z) f(t)=\int_{-\infty}^{\infty} K(t, s ; z) f(s) d s
$$

Let $E(\circ)$ be the spectral projection corresponding to $L_{0,1}$. By a well-known formula [7, p. 1202]

$$
\left(E\left[\lambda_{0}, \lambda\right] u, u\right)=\lim _{\varepsilon \rightarrow 0}+\frac{1}{\pi} \int_{\lambda_{0}}^{\lambda} \operatorname{Im} \int_{R \times R} K(t, s ; \mu+i \varepsilon) d t d s d \mu .
$$

Thus, as in the proof of Theorem 1 of $[2, \mathrm{I}]$ it is enough to show that $K(t, s ; z)$ can be extended continuously to $R \times R \times \Omega(I)$.

Applying our asymptotic lemmas 3.1, 3.2 and the remarks in [2, I] we have for $K$ the following explicit formula

$$
K(t, s ; z)= \begin{cases}\frac{y^{+}(t, z) y^{-}(s, z)}{W_{z}\left(y^{+}, y^{-}\right)}, & t \geqslant s \\ \frac{y^{-}(t, z) y^{+}(s, z)}{W_{z}\left(y^{+}, y^{-}\right)}, & t \leqslant s\end{cases}
$$

where $W_{z}\left(y^{+}, y^{-}\right)$is the Wronskian (which depends on $z$ only).

To establish the stated continuity of $K$, all we have to show is that for real $z \in I$, $W_{z}\left(y^{+}, y^{-}\right) \neq 0$ (note the continuity with respect to $z$ of $y^{ \pm}$as stated in $\S 3$ ).

Suppose to the contrary that for some real $z_{0} \in I, W_{z_{0}}\left(y^{+}, y^{-}\right)=0$. Then there exists a constant $\lambda$ such that

$$
g(t)=\lambda y^{+}\left(t, z_{0}\right)=y^{-}\left(t, z_{0}\right), \quad t \in R
$$

Let us compute $W_{z_{0}}(g, \bar{g})$. As $y^{-}\left(t, z_{0}\right)$ is a real function $W_{z_{0}}(g, \bar{g})=W_{z_{0}}\left(y^{-}, y^{-}\right)$ $=0$. On the other hand, using the asymptotic estimates (3.3) we obtain

$$
W_{z_{0}}\left(\lambda y^{+}\left(t, z_{0}\right), \lambda y^{+}\left(t, z_{0}\right)\right)=-2 i|\lambda|^{2} \neq 0 \text {. Q.E.D. }
$$

We next proceed to a more detailed study of the spectrum in $\Lambda$, leading to an eigenfunction expansion theorem. 
LEMMA 4.3. Let $T_{0}$ be any selfadjoint restriction of $L_{0,1}$ to $(-\infty, 0]$. Then

$$
\sigma_{e}\left(T_{0}\right) \cap \Lambda=\varnothing .
$$

In fact, the only possible limit point of $\sigma\left(T_{0}\right) \cap \Lambda$ is $\Gamma$.

Proof. Let $t_{0}>0$. Denote by $T_{1}$ and $T_{2}$ the restrictions of $T_{0}$ to $\left(-\infty,-t_{0}\right)$ and $\left(-t_{0}, 0\right)$, respectively. By Theorem XIII.7.4 of [7] we have $\sigma_{e}\left(T_{0}\right)=\sigma_{e}\left(T_{1}\right) \cup \sigma_{e}\left(T_{2}\right)=$ $\sigma_{e}\left(T_{1}\right)$, because $T_{2}$ has a compact resolvent. Choose $t_{0}$ sufficiently large and notice that by Lemma 3.2 the solution $y^{-}(t, \lambda)$ does not vanish in $\left(-\infty,-t_{0}\right)$, for $\lambda \in \Lambda$. Thus, by a well-known fact about the zeros of eigensolutions of a selfadjoint operator (Theorem XIII.7.40 of [7]) we see that $T_{1}$ is bounded below, with a bound which is greater than or equal to $\Gamma$. Q.E.D.

THEOREM 4.2. Let $E(\circ)$ be the spectral resolution of $L_{0,1}$ in $L^{2}(R)$.

There exists a function $\varphi(x, \lambda)$, continuous on $R \times \Lambda$, such that for every fixed $\lambda \in \Lambda, \varphi(x, \lambda) \in H_{\mathrm{loc}}^{2}(R)$ and

$$
L_{0,1} \varphi(x, \lambda)=\lambda \varphi(x, \lambda)
$$

(here $L_{0,1}$ is regarded as a differential operator). For a compactly supported $f(x) \in$ $L^{2}(R)$ set

$$
(\mathscr{F} f)(\lambda)=\int_{-\infty}^{\infty} f(x) \overline{\varphi(x, \lambda)} d x, \quad \lambda \in \Lambda
$$

Then:

(a) $\mathcal{F}$ can be extended as a unitary map of $E(\Lambda) L^{2}(R)$ onto $L^{2}(\Lambda ; d \lambda)$ which "diagonalizes" $L_{0,1}$ (as an operator in $\left.L^{2}(R)\right)$, i.e.,

$$
\left(\mathscr{F} L_{(0,1)} f\right)(\lambda)=\lambda(\mathscr{F} f)(\lambda) .
$$

(b) If $g(\lambda) \in L^{2}(\Lambda ; d \lambda)$ is compactly supported then

$$
\left(\mathcal{F}^{*} g\right)(\lambda)=\int_{\Lambda} g(\lambda) \varphi(x, \lambda) d \lambda
$$

(c) There exists a continuous and nowhere vanishing function on $\Lambda, d(\lambda)$, such that

$$
\varphi(x, \lambda)=d(\lambda) y^{-}(x, \lambda) .
$$

Furthermore, if condition (3.9) holds and if for a positive integer $m$ condition (3.6) is satisfied then $d(\lambda)$ is m-times differentiable.

Proof. We recall the basic facts about the spectral representation of a second-order selfadjoint ordinary differential operator $A$, with spectral resolution $E_{A}(\circ)$ : For every interval $J \subset R$ there exist two measures $\mu_{1}, \mu_{2}$ on $J, \mu_{2}$ absolutely continuous with respect to $\mu_{1}$, and a unitary map $U$ of $E_{A}(J) L^{2}(R)$ onto $L^{2}\left(J ; \mu_{1}\right) \oplus L^{2}\left(J ; \mu_{2}\right)$ which diagonalizes $A$. $U$ can be represented in terms of kernels, namely,

$$
(U f)_{k}(\lambda)=\int f(x) \overline{W_{k}(x, \lambda)} d x, \quad k=1,2,
$$

for compactly supported $f$, where $W_{k}(x, \lambda)$ satisfies $A W_{k}(x, \lambda)=\lambda W_{k}(x, \lambda), k=1$, 2. $W_{2}$ is linearly independent of $W_{1}$ for $\mu_{2}$-a.e. $\lambda \in J$. (For more details, see [4, §4] and references there.) 
We now apply this general theory to our operator $L_{0,1}$. Applying Lemma 4.3 and the same proof as of Lemma 4.6 in [4] we conclude that $\mu_{2}(\Lambda)=0$ and hence that $L_{0,1}$ has multiplicity 1 over $\Lambda$. Suppressing the indices in the above representation we now have

$$
U f(\lambda)=\int_{-\infty}^{\infty} f(x) \overline{W(x, \lambda)} d x
$$

and

$$
\left(U^{*} g\right)(x)=\int_{\Lambda} g(\lambda) W(x, \lambda) d \mu(\lambda),
$$

where, for every $\lambda \in \Lambda, W(x, \lambda)$ is square-integrable near $-\infty$. Thus, $W(x, \lambda)$ is a scalar multiple of $y^{-}(x, \lambda)$. By Theorem $4.1 \mu(\lambda)$ is absolutely continuous with respect to the Lebesgue measure $d \lambda$. Let $\alpha(\lambda)=d \mu / d \lambda$ be the Radon-Nikodým derivative of $\mu$ and set

$$
\varphi(x, \lambda)=\alpha(\lambda)^{1 / 2} W(x, \lambda) .
$$

It now follows immediately that the map defined by (4.5) satisfies (4.6)-(4.7). Also note that since $L_{0,1}$ is a real operator it follows that $\varphi(x, \lambda)$ is real.

We now prove $(\mathrm{c})$.

Let $u$ be a continuous compactly supported function. Formula (4.2) implies

$$
\frac{d}{d \lambda}(E(\lambda) u, u)=\frac{1}{\pi} \operatorname{Im} \int_{R \times R} K(t, s ; \lambda) u(s) \overline{u(t)} d s d t .
$$

On the other hand, by the representation (4.5)-(4.6):

$$
\frac{d}{d \lambda}(E(\lambda) u, u)=\int_{R \times R} u(s) \overline{u(t)} \overline{\varphi(s, \lambda)} \varphi(t, \lambda) d s d t .
$$

From (4.9)-(4.10) we conclude

$$
\pi|\varphi(t, \lambda)|^{2}=\operatorname{Im} K(t, t ; \lambda) .
$$

Obviously, a multiplication of $\varphi(t, \lambda)$ by a "sign function" $\nu(\lambda)= \pm 1$ does not change the representation (4.5)-(4.6). Hence we can redefine $\varphi$ so that

$$
\pi^{1 / 2} \varphi(t, \lambda)=[\operatorname{Im} K(t, t ; \lambda)]^{1 / 2} .
$$

By the discussion following formula (4.3) it follows clearly that $K(t, t ; \lambda)$ is continuous in $\lambda$, so that (4.11) implies that $\varphi(t, \lambda)$ is continuous in $\lambda$. Furthermore, since both $y^{ \pm}(t, \lambda) \neq 0$ for every $\lambda \in \Lambda$, it follows from (4.3) that

$$
\operatorname{Im} K(t, t ; \lambda) \neq 0 \text { for every } \lambda \in \Lambda,
$$

and so also $\varphi(t, \lambda) \neq 0$ and the representation (4.8) is justified.

Finally, if condition (3.6) is satisfied for a certain $m$ (and (3.9) holds), then by Lemma 3.3 both $y^{ \pm}(t, \lambda)$ and their first derivatives are $m$-times differentiable with respect to $\lambda$. This is therefore true for $K(t, t ; \lambda)$ for every fixed $t \in R$, and by (4.11)-(4.12) it follows that $\varphi(t, \lambda)$ is also $m$-times differentiable with respect to $\lambda$ (note that by standard theorems about ordinary differential equations it suffices to prove the statement for one fixed value of $t$ ). Q.E.D. 
COROLlaRY 4.1. The operators $-\Delta+V\left(x_{1}\right)$, where $V$ satisfies the assumptions of $\S 2$, are all unitarily equivalent over $\Lambda=(-\infty, \Gamma)$. In particular, they are all purely absolutely continuous there.

Proof. It is obviously sufficient to prove the statement for the corresponding operators $L_{0,1}$ in the decomposition (4.1). In the process of the proof of the preceding theorem it was shown that $L_{0,1}$ is of multiplicity 1 over $\Lambda$. Also, it follows easily from formulas (4.9) and (4.12) that $d E_{\lambda}$ and the Lebesgue measure $d \lambda$ are absolutely continuous with respect to each other. This is enough to ensure the asserted unitary equivalence.

Corollary 4.2. Let $V_{S}(t)=O\left(|t|^{-1-\varepsilon+\alpha / 2}\right)$ as $|t| \rightarrow \infty$ for some $\varepsilon>0$ and set

$$
T_{\alpha}=-\Delta-\left(\operatorname{sgn} x_{1}\right) \cdot\left|x_{1}\right|^{\alpha}+V_{S}\left(x_{1}\right), \quad 0<\alpha \leqslant 2,
$$

then the spectrum of $T_{\alpha}$ in $L^{2}\left(R^{n}\right)$ is $(-\infty, \infty)$ and is purely absolutely continuous. Furthermore, $T_{\alpha}$ is unitarily equivalent to the Stark Hamiltonian $-\Delta-x_{1}$, which in turn is unitarily equivalent to $-\Delta^{\prime}-x_{1}$, where $\Delta^{\prime}$ is the Laplacian with respect to the $(n-1)$ variables $x^{\prime}$ orthogonal to $x_{1}$.

Proof. Everything follows from the preceding corollary, except perhaps for the last statement. But the unitary equivalence of $-\Delta-x_{1}$ and $-\Delta^{\prime}-x_{1}$ follows from an explicit representation given in [1], or else from the unitary equivalence of $-\left(d^{2} / d x^{2}\right)-x$ in $L^{2}\left(R_{x}\right)$ and the multiplication by $\lambda$ in $L^{2}\left(R_{\lambda}\right)$, which is a simple consequence of Theorem 4.2 above.

In particular, the corollary allows for Coulomb-like perturbations of the Stark $(\alpha=1)$ potential. Thus, it provides an extension of the results of [14].

V. Existence of wave operators. Let $L, L_{0}$ be the operators defined by (2.2), where $V_{p}(x)$ is a real potential and $L$ is any selfadjoint realization in $L^{2}\left(R^{n}\right)$ of $-\Delta+$ $V\left(x_{1}\right)+V_{p}(x) / C_{0}^{\infty}\left(R^{n}\right)$. As is well known, the wave-operators associated with $L$, $L_{0}$, are defined as the strong limits

$$
W_{ \pm}=s-\lim _{t \rightarrow \pm \infty} e^{i t L} e^{-i t L_{0}}
$$

if they exist. We shall work with a local modification of this definition, namely, we shall prove, under certain conditions on $V_{p}(x)$, the existence of the strong limits

$$
W_{ \pm}(I)=s-\lim _{t \rightarrow \pm \infty} e^{i t L} e^{-i t L_{0}} E(I) .
$$

Here $I \subseteq \Lambda=(-\infty, \Gamma)$ (see definition (2.3)) is a compact interval and $E(\circ)$ is the spectral family associated with $L_{0}$, which has a unique selfadjoint realization by Lemma 4.1.

Obviously if $\Lambda=R$ and the limits (5.1) exist for all $I$, it follows that the limits $W_{ \pm}$ also exist.

Henceforth we shall always assume that condition (3.9) is fulfilled.

For the transformation (4.5), we denote $\tilde{f}(\lambda) \equiv(\mathscr{F} f)(\lambda)$.

Let $\psi(x) \in L^{2}\left(R^{n}\right), \psi(x)=\psi_{1}\left(x_{1}\right) \psi_{2}\left(x^{\prime}\right), x=\left(x_{1}, x^{\prime}\right)$. Using the decomposition (4.1) we have

$$
e^{-i t L_{0}} \psi=e^{-i t L_{0,1}} \psi_{1} \cdot e^{+i t \Delta^{\prime}} \psi_{2}
$$


Let $\hat{\psi}_{2}$ denote the Fourier transform of $\psi_{2}$ (with respect to the $(n-1)$ variables $\left.x^{\prime}\right)$.

Then

$$
\hat{\psi}_{2} \in C_{0}^{\infty}\left(R_{\xi}^{n-1}\right) \Rightarrow \psi_{2} \in S\left(R_{x^{\prime}}^{n-1}\right),
$$

where $S$ is the Schwartz space of rapidly decaying functions.

Also, if $\tilde{\psi}_{1}(\lambda)$ is bounded compactly supported in $\Lambda$, then by (4.6)

$$
\left(e^{-i t L_{0,1}} \psi_{1}\right)(\lambda)=e^{-i t \lambda} \tilde{\psi}_{1}(\lambda)
$$

and by (4.8) and Lemma 3.2(b) $e^{-i t L_{0,1}} \psi_{1}\left(x_{1}\right)$ decays exponentially as $x_{1} \rightarrow-\infty$.

In what follows we shall find it convenient to denote

$$
\psi_{1}^{t}\left(x_{1}\right)=\exp \left(-i t L_{0,1}\right) \psi_{1}\left(x_{1}\right), \quad \psi_{2}^{t}\left(x^{\prime}\right)=\exp \left(+i t \Delta^{\prime}\right) \psi_{2}\left(x^{\prime}\right) .
$$

By unitarity, it is enough to establish the existence of the limits (5.1) for a fundamental set in $E(\Lambda) L^{2}\left(R^{n}\right)$,

$$
Q=\left\{\psi / \psi(x)=\psi_{1}\left(x_{1}\right) \psi_{2}\left(x^{\prime}\right), \tilde{\psi}_{1}(\lambda) d(\lambda) \in C_{0}^{\infty}(\Lambda), \hat{\psi}_{2}\left(\xi^{\prime}\right) \in C_{0}^{\infty}\left(R_{\xi^{\prime}}^{n-1}\right)\right\}
$$

where $d(\lambda)$ is given by (4.8).

By the above remarks

$$
\operatorname{Supp} \tilde{\psi}_{1}(\lambda) \subseteq I, \quad \psi \in Q \Rightarrow E(I) \psi \in \overline{\operatorname{Span} Q} .
$$

Thus, the well-known Cook's method (see [13]) is applicable in our case in the following sense.

Let $L$ be any selfadjoint realization of $-\Delta+V\left(x_{1}\right)+V_{p}(x)$ such that $Q \subseteq D(L)$ and

$$
L \psi=\left(-\Delta+V\left(x_{1}\right)\right) \psi+V_{p} \cdot \psi, \quad \psi \in Q,
$$

then the wave-operator $W_{+}(I)\left(W_{-}(I)\right)$ exists if, for every $\psi \in Q$, there is a $t_{0}$ such that

$$
\left\|V_{p} \exp \left(-i t L_{0}\right) \psi\right\| \in L^{1}\left(\left(t_{0}, \infty\right) ; d t\right)\left(L^{1}\left(\left(-\infty, t_{0}\right) ; d t\right)\right) .
$$

As a simple application we have

LEMMA 5.1. Let $n>3$ and $V_{p}(x) \in L^{2}\left(R^{n}\right)$. Then $W_{ \pm}(I)$ exist for every $I \subseteq \Lambda$.

PROof. By a standard estimate for the free Hamiltonian (see [11])

$$
\left|\psi_{2}^{t}\left(x^{\prime}\right)\right| \leqslant C|t|^{-(n-1) / 2} .
$$

Thus $\left\|V_{p} \exp \left(-i t L_{0}\right) \psi\right\| \leqslant C\left\|V_{p}\right\| t^{-(n-1) / 2}$. Q.E.D.

In the above proof, no use has been made of the special features of the eigenfunction expansion associated with $L_{0,1}$, especially the exponential decay at $-\infty$. In fact, using the notation $\chi(A)$ for the characteristic function of $A \subseteq R^{n}$, we have

Lemma 5.2. Assume that for some $N>0$,

$$
(1+|x|)^{-N} V_{p}(x) \in L^{2}\left(R^{n}\right),
$$


and let $x_{1}^{0}$ be fixed and $\psi \in Q$. Then for every $M$ there exists a constant $C_{M}$ (which depends on $\psi, V_{p}$ ) and $t_{0}$ such that

$$
\left\|\chi\left(x_{1} \leqslant x_{1}^{0}\right) V_{p}(x) \exp \left(-i t L_{0}\right) \psi\right\| \leqslant C_{M}|t|^{-M}, \quad|t| \geqslant t_{0} .
$$

Proof. Using Theorem 4.2 we have

$$
\psi_{1}^{t}\left(x_{1}\right)=\int_{\Lambda} e^{-i t \lambda} \tilde{\psi}_{1}(\lambda) d(\lambda) y^{-}\left(x_{1}, \lambda\right) d \lambda .
$$

Since $\tilde{\psi}_{1}(\lambda) d(\lambda) \in C_{0}^{\infty}(\Lambda)$ we can integrate by parts and taking the estimates (3.10) into account we see that for every positive integer $r$ there exists a constant $c_{r}$ such that

$$
\left|\psi_{1}^{t}\left(x_{1}\right)\right| \leqslant c_{r}\left(1+|t|+\left|x_{1}\right|\right)^{-r}, \quad x_{1} \leqslant x_{1}^{0} .
$$

By a standard stationary phase argument (see [13]) there exists an $R>0$ such that for every positive integer $s$ there exists a constant $c_{s}$ and

$$
\left|\psi_{2}^{t}\left(x^{\prime}\right)\right| \leqslant c_{s}\left(1+\left|x^{\prime}\right|+|t|\right)^{-s}, \quad\left|x^{\prime}\right| \geqslant R|t|,
$$

and

$$
\left|\psi_{2}^{t}\left(x^{\prime}\right)\right| \leqslant c_{2} \quad \text { for all } x^{\prime} .
$$

Combining (5.8)-(5.9) with assumption (5.6) we obtain (5.7). Q.E.D.

Since the behavior of the eigenfunctions $\varphi\left(x_{i}, \lambda\right)$ is oscillatory for large positive $x_{1}$, the conditions imposed there on $V_{p}$ will naturally be stronger than (5.6).

To simplify notation, we shall work the case $n=3$. As is seen from Lemma 5.1 and its proof the cases $n>3$ are always simpler.

We shall also need the following modification of the eigenfunction expansion (4.7).

We note that by Lemma 3.1 , the functions $y^{+}(t, \lambda), \overline{y^{+}(t, \lambda)}, \lambda \in \Lambda$, are independent solutions of equation (3.1). Hence,

$$
y^{-}(t, \lambda)=\theta_{1}(\lambda) y^{+}(t, \lambda)+\theta_{2}(\lambda) \overline{y^{+}(t, \lambda)}, \quad \lambda \in \Lambda .
$$

If $y^{+}(t, \lambda)$ is $m$-times differentiable with respect to $\lambda$, then so are $\theta_{1}(\lambda), \theta_{2}(\lambda)$.

We can now represent

$$
\psi_{1}^{t}\left(x_{1}\right)=\int_{\Lambda} e^{-i t \lambda} \tilde{\psi}_{1}(\lambda) d(\lambda)\left(\theta_{1}(\lambda) y^{+}\left(x_{1}, \lambda\right)+\theta_{2}(\lambda) y^{+}\left(x_{1}, \lambda\right)\right) d \lambda
$$

In order to allow for a good asymptotic evaluation of $\psi_{1}^{t}\left(x_{1}\right)$ we assume that the following condition holds true:

(A) Condition (3.6) is satisfied with $m=2$.

Using the notation

$$
\eta(t, \lambda)=(1+a(t, \lambda))\left(\lambda-V_{L}(t)\right)^{1 / 2}
$$

for the exponent in (3.3), Lemma 3.3(a) implies that under condition (A)

$$
y^{+}(t, \lambda)=\eta(t, \lambda)^{-1 / 2}\left[\exp \left(i \int_{t_{0}}^{t} \eta(s, \lambda) d s\right)+r^{+}(s, \lambda)\right] \text {, }
$$


where $r^{+}(s, \lambda)$ is twice differentiable with respect to $\lambda$ and

$$
\left|r^{+}(t, \lambda)\right|+\left|\frac{d}{d \lambda} r^{+}(t, \lambda)\right|+\left|\left(\frac{d}{d \lambda}\right)^{2} r^{+}(t, \lambda)\right|=O(1),
$$

for $t_{0} \leqslant t<\infty, \lambda \in I$.

Another growth condition on $V_{L}$ which will be used below is the following:

(B) For every $\varepsilon>0$ let $g_{\varepsilon}(t)=\sup _{x_{1}>\varepsilon t}\left(1+\left|V_{L}\left(x_{1}\right)\right|\right)^{-1 / 4}$.

Then there exists a sufficiently large $t_{0}$ such that $t^{-1} g_{\varepsilon}(t) \in L^{1}\left(\left(t_{0}, \infty\right) ; d t\right)$.

LEMMA 5.3. Let $V_{L}$ satisfy conditions (A) and (B) and let $V_{p} \in L^{2}\left(R^{3}\right)$. Then, for $x_{1}^{0}$, $t_{0}$ sufficiently large we have

$$
\left\|\chi\left(x_{1} \geqslant x_{1}^{0}\right) V_{p} \exp \left(-i t L_{0}\right) E(I) \psi\right\| \in L^{1}\left(\left(-\infty,-t_{0}\right) \cup\left(t_{0}, \infty\right) ; d t\right)
$$

for $\psi \in Q$, Supp $\tilde{\psi}_{1}(\lambda) \subseteq I \subseteq \Lambda$.

Proof. We prove only for $t_{0} \leqslant t<\infty$, the interval $-\infty<t \leqslant-t_{0}$ being exactly the same.

Using condition (A) and the representations (5.11), (5.13) we have, if Supp $\tilde{\psi}_{1}(\lambda)$ $\subseteq I$ :

$$
\begin{aligned}
\psi_{1}^{t}\left(x_{1}\right)= & \int_{I} e^{-i t \lambda} \tilde{\psi}_{1}(\lambda) d(\lambda) \theta_{1}(\lambda) \eta\left(x_{1}, \lambda\right)^{-1 / 2} \exp \left(i \int_{x_{1}^{0}}^{x_{1}} \eta(s, \lambda) d s\right) d \lambda \\
& +\int_{I} e^{-i t \lambda} \tilde{\psi}_{1}(\lambda) d(\lambda) \theta_{2}(\lambda) \eta\left(x_{1}, \lambda\right)^{-1 / 2} \exp \left(i \int_{x_{1}^{0}}^{x_{1}}-\eta(s, \lambda) d s\right) d \lambda \\
& +\int e^{-i t \lambda} \psi_{1}(\lambda) d(\lambda) \eta\left(x_{1}, \lambda\right)^{-1 / 2}\left(\theta_{1}(\lambda) r^{+}\left(x_{1}, \lambda\right)+\theta_{2}(\lambda) \overline{r^{+}\left(x_{1}, \lambda\right)}\right) d \lambda \\
= & J_{1}+J_{2}+J_{3} .
\end{aligned}
$$

Integrating in $J_{3}$ by parts twice and using (5.14) we get

$$
\left|J_{3}\right| \leqslant C t^{-2}
$$

hence

$$
\left\|V_{p} J_{3} \psi_{2}^{t}\left(x^{\prime}\right)\right\| \leqslant C\left\|V_{p}\right\| t^{-2} .
$$

To estimate $J_{2}$, we note that by Lemma 3.1(d) and definitions (3.5), (5.12), there exist constants $c_{1}, c_{2}$ such that

$$
c_{1} w\left(x_{1}\right) \leqslant\left|\frac{d}{d \lambda} \int_{x_{1}^{0}}^{x_{1}} \eta\left(x_{1}, \lambda\right)\right| \leqslant c_{2} w\left(x_{1}\right), \quad x_{1}^{0} \leqslant x_{1}<\infty, \lambda \in I .
$$

Integrating $N$ times by parts we obtain

$$
\left|J_{2}\right| \leqslant C_{N}\left(w\left(x_{1}\right)+t\right)^{-N} \eta(x, \lambda)^{-1 / 2},
$$

hence

$$
\left\|V_{p} J_{2} \psi_{2}^{t}\left(x^{\prime}\right)\right\| \leqslant C_{N}\left\|V_{p}\right\|(1+t)^{-N} .
$$


In order to estimate $J_{1}$ we note that by (3.5) $\left|w\left(x_{1}\right)\right| \leqslant x_{1}$. Therefore, by (5.18) the phase function $-i t \lambda+i \int_{x \rho}^{x_{1}} \eta(s, \lambda) d s$ has no stationary point $\lambda \in \operatorname{Supp} \tilde{\psi}_{1}$ if $x_{1} \leqslant \varepsilon t$, where $\varepsilon>0$ is sufficiently small. Hence

$$
\left|\chi\left(x_{1} \leqslant \varepsilon t\right) J_{1}\right| \leqslant C_{N}\left(w\left(x_{1}\right)+t\right)^{-N} w^{\prime}\left(x_{1}\right)^{1 / 2},
$$

and using (5.12) and the definition of $g_{\varepsilon}(t)$ we have

$$
\left|\chi\left(x_{1} \geqslant \varepsilon t\right) J_{1}\right| \leqslant C g_{\varepsilon}(t) \text {. }
$$

Estimating now $\psi_{2}^{t}\left(x^{\prime}\right)$ by (5.5) (with $n=3$ ) we conclude

$$
\left\|V_{p} J_{1} \psi_{2}^{t}\left(x^{\prime}\right)\right\| \leqslant C\left\|V_{p}\right\|\left[(1+t)^{-N}+t^{-1} g_{\varepsilon}(t)\right] \text {. }
$$

Putting together inequalities (5.17), (5.19), (5.23) and noting condition (B) the lemma follows. Q.E.D.

In Lemma 5.3 we have imposed a "global" restriction on the perturbation $V_{p}$. Our next lemma shows how one can replace this requirement by a growth condition as $x_{1} \rightarrow+\infty$. In fact, a suitable decay on $x_{1} \rightarrow+\infty$ can compensate for an unbounded behavior in the $x^{\prime}$ directions. To gain clarity, we make no attempt at obtaining the best possible results along this line.

We shall now assume that, for some $\delta>0$

$$
V_{L}\left(x_{1}\right) \in \bigcap_{k=1}^{\infty} B(k, \delta ;+\infty)
$$

(see definition (2.1)) and that $V_{S}\left(x_{1}\right)$ decays exponentially as $x_{1} \rightarrow+\infty$.

Thus, condition (3.1) is fulfilled with any integer $m$. In fact, since $|w(t)| \leqslant t$ by (3.5), we have for every two positive integers $m, N$ a positive integer $k_{m, N}$ such that for $q(\xi)$ defined by (3.6) with $k=k_{m, N}$

$$
|q(\xi)| \leqslant C_{N} \xi^{-N} \text {. }
$$

LEMMA 5.4. Assume that $V_{L}$ satisfies condition (5.23). Suppose also that

$$
\begin{gathered}
\left|V_{p}\left(x_{1}, x^{\prime}\right)\right| \leqslant C\left(1+x_{1}\right)^{-s}\left(1+\left|x^{\prime}\right|\right)^{r}, \quad x_{1} \geqslant x_{1}^{0}, \\
\left(1+x_{1}\right)^{-s}\left(1+\left|V_{L}\left(x_{1}\right)\right|\right)^{-1 / 4} w\left(x_{1}\right)^{r_{1}} \in L^{2}\left(\left(x_{1}^{0}, \infty\right) ; d x_{1}\right),
\end{gathered}
$$

where $x_{1}^{0}$ is sufficiently large, $s>0, r \geqslant 0$ and $r_{1}>r+1 / 2$.

Then for $t_{0}$ sufficiently large and $\psi \in Q$, supp $\tilde{\psi}_{1} \subseteq I \subseteq \Lambda$

$$
\left\|\chi\left(x_{1} \geqslant x_{1}^{0}\right) V_{p} \exp \left(-i t L_{0}\right) E(I) \psi\right\| \in L^{1}\left(\left(-\infty,-t_{0}\right) \cup\left(t_{0}, \infty\right) ; d t\right) .
$$

Proof. Again we prove for $t \geqslant t_{0}$. For $\psi_{1}^{t}\left(x_{1}\right)$ we use the representation (5.16).

Observe that by inequality (5.9) and (5.25)

$$
\left|\chi\left(\left|x^{\prime}\right| \geqslant R t\right) V_{p} \psi_{1}^{t} \psi_{2}^{t}\right| \leqslant C_{M}\left(1+x_{1}\right)^{-s}\left(1+\mid V_{L}\left(x_{1}\right)\right)^{-1 / 4}\left(1+\left|x^{\prime}\right|+t\right)^{-M},
$$

for every positive integer $M$. By (5.26) the right-hand side of (5.28) is square-integrable and its norm is $d t$-summable. Thus, it suffices to prove (5.27) for $\left|x^{\prime}\right| \leqslant R t$, where by (5.25)

$$
\left|V_{p}\left(x_{1}, x^{\prime}\right)\right| \leqslant C(1+t)^{r}\left(1+x_{1}\right)^{-s}
$$


By (5.24) and (3.8) we can assume that $r^{+}\left(x_{1}, \lambda\right)$ and its first $m$ derivatives with respect to $\lambda$ are bounded by $C\left(1+x_{1}\right)^{-1}$. Taking $m$ large enough and integrating in $J_{3}$ by parts $m$-times we obtain

$$
\left|J_{3}\right| \leqslant C t^{-m}\left(1+\left|V_{L}\left(x_{1}\right)\right|\right)^{-1 / 4}
$$

so that by (5.29), with $m>r+2$

$$
\left|V_{p} J_{3}\right| \leqslant C t^{-2}\left(1+x_{1}\right)^{-s}\left(1+\left|V_{L}\left(x_{1}\right)\right|\right)^{-1 / 4},
$$

which implies, by (5.26)

$$
\left\|\chi\left(x_{1} \geqslant x_{1}^{0},\left|x^{\prime}\right| \leqslant R t\right) V_{p} J_{3} \psi_{2}^{t}\right\| \leqslant C t^{-2} .
$$

The estimate for $J_{2}$ is also simple. In fact, all we have to do is replace the above estimate for $\left|J_{3}\right|$ by the inequality for $\left|J_{2}\right|$ just preceding (5.19).

The estimate for $J_{1}$ is more subtle. We first show that

$$
\left(\left|1+V_{L}\left(x_{1}\right)\right|\right)^{1 / 4}\left|\psi_{1}^{t}\left(x_{1}\right)\right| \leqslant c t^{-1 / 2}, \quad t \geqslant t_{0} .
$$

This is proved by a stationary phase argument as follows: Consider the phase function

$$
\gamma(\lambda)=-i t \lambda+i \int_{x_{1}^{0}}^{x_{1}} \eta(s, \lambda) d s \quad(\text { see }(5.12)) .
$$

By (5.18) and $\left|w\left(x_{1}\right)\right| \leqslant\left|x_{1}\right|, \gamma$ has no stationary point for $x_{1} \leqslant \varepsilon t, \varepsilon>0$ sufficiently small. Also, by Lemma 3.1(d)

$$
\left(\frac{d}{d \lambda}\right)^{2} \gamma(\lambda)=i \int_{x_{1}^{0}}^{x_{1}} \frac{\partial^{2}}{\partial \lambda^{2}} \eta(s, \lambda) d s \neq 0, \quad \lambda \in I .
$$

We now have all that is needed to obtain (5.31) (see [13, Theorem XI.15]).

Combining (5.29) and (5.31) (for $\left|x^{\prime}\right| \leqslant R t$ )

$$
\left|V_{p} J_{1} \psi_{1}^{t}\right| \leqslant C \cdot\left(1+x_{1}\right)^{-s}\left(1+\left|V_{L}\right|\right)^{-1 / 4}(1+t)^{r-1 / 2} \text {. }
$$

Integrating in $J_{1} N$-times by parts we get

$$
\left|V_{p} J_{1} \psi_{1}^{t}\right| \leqslant C \cdot \frac{1}{t^{N}}\left(1+x_{1}\right)^{-s} w\left(x_{1}\right)^{N}\left(1+\left|V_{L}\right|\right)^{-1 / 4}(1+t)^{r-1 / 2} .
$$

From (5.32)-(5.33) we obtain, with $0<\varepsilon<1$,

$$
\left|V_{p} J_{1} \psi_{1}^{t}\right| \leqslant C(1+t)^{r-(1 / 2)-\varepsilon N} w\left(x_{1}\right)^{N \varepsilon}\left(1+x_{1}\right)^{-s}\left(1+\left|V_{L}\right|\right)^{-1 / 4} .
$$

Take $\varepsilon>(r+1 / 2) / N$ but such that $N \varepsilon \leqslant r_{1}$. Then, with some $\beta>0$ we have

$$
\left|V_{p} J_{1} \psi_{1}^{t}\right| \leqslant C(1+t)^{-1-\beta} w\left(x_{1}\right)^{r_{1}}\left(1+x_{1}\right)^{-s}\left(1+\left|V_{L}\right|\right)^{-1 / 4} \text {. }
$$

By (5.26) we conclude finally

$$
\left\|\chi\left(\left|x^{\prime}\right| \leqslant R t, x_{1} \geqslant x_{1}^{0}\right) V_{p} \exp \left(-i t L_{0}\right) E(I) \psi\right\| \leqslant C t^{-1-\beta} \text {. Q.E.D. }
$$

We are now in a position to state the following theorem. 
THEOREM 5.1. Let $Q$ be the set defined by (5.4) and let $L$ be any selfadjoint realization in $L^{2}\left(R^{3}\right)$ of $-\Delta+V\left(x_{1}\right)+V_{p}(x)$ such that $Q \subseteq D(L)$.

Let $I \subseteq \Lambda$ be a compact interval (where $\Lambda=(-\infty, \Gamma)$ is defined by (2.3)). Then the limits $W_{ \pm}(I)$ ( see (5.1)) exist in either one of the following cases:

(a) (1) For $w(y)=\int_{y_{0}}^{y}\left(1+\left|V_{L}(s)\right|\right)^{-1 / 2} d s, y \geqslant y_{0}$,

$$
\begin{aligned}
w(y)^{2}\left\{\left|V_{S}(y)\right|\left(1+\left|V_{L}(y)\right|\right)^{-1 / 2}+\left(1+\left|V_{L}(y)\right|\right)^{-k+1 / 2}(1+y)^{-2 k \delta}\right\} & \in L^{1}\left(y_{0}, \infty\right) .
\end{aligned}
$$

(2) For every $\varepsilon>0$, if $g_{\varepsilon}(y)=\sup _{s>\varepsilon y}\left(1+\left|V_{L}(s)\right|\right)^{-1 / 4}$ then $y^{-1} g_{\varepsilon}(y) \in$ $L^{1}\left(y_{0}, \infty\right)$.

(3) $V_{p}(x) \in L^{2}\left(R^{3}\right)$.

(b) (1) $V_{L} \in \cap_{k=1}^{\infty} B(k, \delta ;+\infty)$ for some $\delta>0$.

(2) $(1+y)^{-s}\left(1+\left|V_{L}(y)\right|\right)^{-1 / 4} w(y)^{r_{1}} \in L^{2}\left(y_{0}, \infty\right)$ where $s>0, r_{1}>r+1 / 2$, $r \geqslant 0$, and,

(3) $V_{p}\left(x_{1}, x^{\prime}\right) \leqslant C\left(1+x_{1}\right)^{-s}\left(1+\left|x^{\prime}\right|\right)^{r}, x_{1} \geqslant y_{0}$.

(4) For some integer $N:(1+|x|)^{-N} V_{p}(x) \in L^{2}\left(R^{3}\right)$.

Proof. This is just a summary of the above lemmas, using Cook's method. For negative $x_{1}$ we use Lemma 5.2 in both cases. Case (a) now follows from Lemma 5.3 whereas case (b) is discussed in Lemma 5.4.

Observe that if the conditions of both cases are satisfied by $V_{L}$, we can allow $V_{p}=V_{a}+V_{b}$, where the two terms satisfy respectively the conditions imposed on $V_{p}$ in cases (a), (b).

Note that in case $L$ is defined in $L^{2}\left(R^{n}\right), n>3$, the assumptions on the potentials can be relaxed in view of Lemma 5.1.

REMARK. We have discussed in this section only multidimensional perturbations of $L_{0}$. However, if $V_{p}$ depends on $x_{1}$ only we can apply our eigenfunction expansion (Theorem 4.2) in order to give an explicit representation of the wave-operators. In fact, this can be done using the method of Green-Lanford ([8], and see also the modifications in $[5,6])$. In this case, the method yields not only existence, but also completeness of the wave-operators (see $\$ 5$ in [4]). Basically, the estimates needed are similar to the ones used above and similar conditions are required, but we shall not pursue the details.

Also, our methods can be applied to the case that $V_{L}$ is spherically symmetric. In this case the proof of the existence of wave operators for nonspherically symmetric perturbations proceeds along the same lines as in [5]. Note that our general conditions (\$2) on $V_{L}$ are then satisfied if $V_{L}(r)=r^{-\beta} \sin \left(r^{\alpha}\right), \beta>0, \alpha<1$, so that we can somewhat extend the range of values in [5].

VI. Stark-like potentials. The previous results were stated in rather general terms. In this section we specialize to Stark-like operators, i.e.

$$
T_{\alpha}=-\Delta-\left(\operatorname{sgn} x_{1}\right)\left|x_{1}\right|^{\alpha}+V_{S}\left(x_{1}\right), \quad 0<\alpha \leqslant 2 .
$$

The Stark effect is given by $\alpha=1$. 
Under the conditions imposed on $V_{S}$ in $\S I I$ it follows that $T_{\alpha} \mid C_{0}^{\infty}\left(R^{n}\right)$ is essentially selfadjoint. We retain the notation $T_{\alpha}$ for its unique selfadjoint extension in $L^{2}\left(R^{n}\right)$.

Corollary 4.2 has already given the absolute continuity of $T_{\alpha}$ over $(-\infty, \infty)$, assuming a suitable decay condition on $V_{S}$. As a matter of fact, $T_{\alpha}$ is unitarily equivalent to a multiplication operator in $L^{2}\left(R^{n}\right)$ via the following eigenfunction expansion theorem.

THEOREM 6.1. Let $V_{S}(t)=O\left(|t|^{-1-\varepsilon+\alpha / 2}\right)$ as $|t| \rightarrow \infty$ for some $\varepsilon>0$.

There exists a function $\psi(x, k)$, defined and continuous on $R_{x}^{n} \times R_{k}^{n}$, and such that

(a) for every fixed $k \in R^{n}, \psi(x, k) \in H_{\text {loc }}^{2}\left(R_{x}^{n}\right)$ and

$$
T_{\alpha} \psi=\left(k_{1}+\left|k^{\prime}\right|^{2}\right) \psi, \quad k=\left(k_{1}, k^{\prime}\right),
$$

(b) $f o r f(x) \in C_{0}^{\infty}\left(R^{n}\right)$ define the transformation

$$
(\mathscr{F} f)(k)=\int f(x) \overline{\psi(x, k)} d x .
$$

Then $\mathcal{F}$ can be extended as a unitary map of $L^{2}\left(R_{x}^{n}\right)$ onto $L^{2}\left(R_{k}^{n}\right)$. Its adjoint is then given by

$$
\left(\mathscr{F}^{*} g\right)(x)=\text { 1.i.m. } \int g(k) \psi(x, k) d k \text {. }
$$

(c) Let $E(\cdot)$ be the spectral measure associated with $T_{\alpha}$. If $I \subseteq(-\infty, \infty)$

$$
\mathscr{F}(E(I) f)=\chi\left(k_{1}+\left|k^{\prime}\right|^{2} \in I\right)(\mathscr{F} f)(k) .
$$

In particular, for $f \in \mathscr{Q}\left(T_{\alpha}\right)$

$$
\mathscr{F}\left(T_{\alpha} f\right)(k)=\left(k_{1}+\left|k^{\prime}\right|^{2}\right)(\mathscr{F} f)(k) .
$$

(d) Suppose that for some positive integer $m$ and some $\varepsilon>0$

$$
V_{S}(t)= \begin{cases}O\left(t^{-1-\varepsilon-m+(\alpha / 2)(m+1)}\right) & \text { as } t \rightarrow+\infty, 0<\alpha<2, \\ O\left((\log t)^{-m} t^{-\varepsilon}\right) & \text { as } t \rightarrow+\infty, \alpha=2,\end{cases}
$$

then $\psi(x, k)$ is m-times continuously differentiable in $k$. In particular, if $V_{S}(t)$ decay exponentially as $t \rightarrow+\infty$ then

$$
\psi(x, k) \in C^{\infty}\left(R_{k}^{n}\right) .
$$

Proof. The theorem is in fact a straightforward adaptation of Theorem 4.2. The functions $\psi(x, k)$ can be defined as

$$
\psi(x, k)=\varphi\left(x_{1}, k_{1}\right) \exp \left(-i k^{\prime}, x^{\prime}\right)
$$

where $\varphi$ satisfies (4.4).

Parts (a)-(c) then follow immediately. As for part (d) of our theorem, observe that in our case the function $w$ given by (3.5) can be taken as

$$
w(t)= \begin{cases}t^{1-\alpha / 2}, & 0<\alpha<2 \\ \log t, & \alpha=2\end{cases}
$$


Also, the $k$ in (3.6) can be taken arbitrarily large (with $\delta=1$ ). Thus condition (6.6) is a restatement of (3.6) and the statement follows from Theorem 4.2(c). Q.E.D.

In the next theorem we discuss the existence of wave operators for the pair $T_{\alpha}$, $T=T_{\alpha}+V_{p}(x)$ in $L^{2}\left(R^{3}\right)$. We set

$$
Q=\left\{f \in L^{2}\left(R_{x}^{3}\right) / \mathscr{F} f \in C_{0}^{\infty}\left(R_{k}^{3}\right)\right\}
$$

$Q$ is dense in $L^{2}\left(R^{3}\right)$ and for every $t, \exp \left(-i t T_{\alpha}\right) Q \subseteq Q$. It will be clear from our conditions that $V_{p} f$ is well defined for every $f \in Q$. The operator $T$ is then understood to be any selfadjoint extension of $\left(T_{\alpha}+V_{p}\right) / Q$.

THEOREM 6.2. Assume that $V_{S}\left(x_{1}\right)$ satisfies condition (6.6) with $m=2$. Suppose also that for some $N,(1+|x|)^{-N} V_{p}(x) \in L^{2}\left(R^{3}\right)$ and that $V_{p}$ can be decomposed as $V_{p}=V_{1}+V_{2}$ where

(a) $V_{1}(x) \in L^{2}\left(R^{3}\right)$.

$$
\left|V_{2}\left(x_{1}, x^{\prime}\right)\right| \leqslant C\left(1+\left|x^{\prime}\right|\right)^{r}\left(1+x_{1}\right)^{-s} \text { for } x_{1} \geqslant 0,
$$

where $r \geqslant 0, s>(r+1)(1-\alpha / 2)$ if $0<\alpha \leqslant 2$.

Then the wave-operators $W_{ \pm}\left(T, T_{\alpha}\right)$ exist.

Proof. In our case the potential $V_{L}$ satisfies the assumptions of both cases (a) and (b) in Theorem 5.1. The condition on $s$ in (b) follows from condition (b)(2) in Theorem 5.1 by taking (6.7) into account.

The special case $\alpha=1$ is the Stark potential and Theorem 6.2 yields the theorem mentioned in the introduction in this case.

COROLlARY 6.1. Under the assumptions of the previous theorem the absolutely continuous part of the spectrum of $T$ contains the whole line $(-\infty, \infty)$.

Appendix. In this appendix we give the proofs of the asymptotic lemmas stated in §III. Lemma 3.1 is taken from [2], where its proof is given in complete detail. Therefore, we shall just give the technical steps involved in the construction of the asymptotic expansion. This will also enable us to sketch briefly the proof of Lemma 3.2, which is similar to that of Lemma 3.1. Finally, Lemma 3.3 is proved by using some of the simplifications obtained in the process of proving the previous lemmas.

Proof of Lemma 3.1. Our equation is

$$
\left(-\frac{d^{2}}{d t^{2}}+V(t)\right) y(t)=z y(t), \quad z \in \Omega(I), I \subseteq \Lambda .
$$

By rearranging $V=V_{L}+V_{S}$ if necessary we may assume that $t$ varies in $\left[t_{0}, \infty\right)$ where $\operatorname{Re} z>V_{L}(t), z \in \Omega(I)$. Thus $\left(z-V_{L}(t)\right)^{\alpha}, \alpha>0$, is well defined, using the principal branch of the logarithm.

We change now both the independent variable $t$ and the dependent variable $y$ as follows:

$$
\begin{aligned}
\xi_{1}(t, z) & =\int_{t_{0}}^{t}\left(z-V_{L}(s)\right)^{1 / 2} d s, \\
y_{1}\left(\xi_{1}\right) & =\left(z-V_{L}(t)\right)^{1 / 4} y(t) .
\end{aligned}
$$


Note that in (A.3) the variables $\xi_{1}$ and $t$ are related via (A.2). This, with $\xi_{1}$ replaced by $\xi_{k}$ will apply also to the equations below. Also note that (A.2) defines a curve $\Gamma_{1}(z)$, parametrized by $t_{0} \leqslant t<\infty$, in the complex plane. Differentiation and integration with respect to $\xi_{1}$ in what follows will be understood as performed along $\Gamma_{1}$. Using these conventions we now set

$$
\begin{aligned}
B_{1}\left(\xi_{1}\right) & =\frac{1}{2} V_{L}^{\prime}(t)\left(z-V_{L}(t)\right)^{-3 / 2} \\
p_{1}\left(\xi_{1}\right) & =-\frac{1}{2} \frac{d}{d \xi_{1}} B_{1}\left(\xi_{1}\right)+\frac{1}{4}\left(B_{1}\left(\xi_{1}\right)\right)^{2} \\
& =-\left[\frac{1}{4} V_{L}^{\prime \prime}(t)\left(z-V_{L}(t)\right)^{-2}+\frac{5}{16} V_{L}^{\prime}(t)^{2}\left(z-V_{L}(t)\right)^{-3}\right], \\
q_{1}\left(\xi_{1}\right) & =V_{S}(t)\left(z-V_{L}(t)\right)^{-1},
\end{aligned}
$$

so that equation (A.1) takes the form

$$
\left[-\left(\frac{d}{d \xi_{1}}\right)^{2}+\left(p_{1}\left(\xi_{1}\right)+q_{1}\left(\xi_{1}\right)\right)\right] y_{1}\left(\xi_{1}\right)=y_{1}\left(\xi_{1}\right) .
$$

We now define successively a sequence of transformations for $j=2, \ldots, k$ as follows:

$$
\xi_{j}(t, z)=\int_{\xi_{j-1}^{0}}^{\xi_{j-1}}\left(1-p_{j-1}(\eta)\right)^{1 / 2} d \eta,
$$

where $\xi_{j-1}^{0}=\xi_{j-1}\left(t_{0}, z\right)$.

$$
\begin{aligned}
& B_{j}\left(\xi_{j}\right)=\frac{1}{2}\left(\frac{d}{d \xi_{j-1}} p_{j-1}\left(\xi_{j-1}\right)\right) \cdot\left(1-p_{j-1}\left(\xi_{j-1}\right)\right)^{-3 / 2} \\
& p_{j}\left(\xi_{j}\right)=-\frac{1}{2} \frac{d}{d \xi_{j}} B_{j}\left(\xi_{j}\right)+\frac{1}{4}\left[B_{j}\left(\xi_{j}\right)\right]^{2}, \\
& q_{j}\left(\xi_{j}\right)=q_{j-1}\left(\xi_{j-1}\right)\left(1-p_{j-1}\left(\xi_{j-1}\right)\right)^{-1}, \\
& y_{j}\left(\xi_{j}\right)=\left(1-p_{j-1}\left(\xi_{j-1}\right)\right)^{1 / 4} y_{j-1}\left(\xi_{j-1}\right) .
\end{aligned}
$$

Note that $\xi_{j}(t, z)$ defines a curve $\Gamma_{j}(z)$ in the complex plane. The integration in (A.5) is carried out along $\Gamma_{j-1}(z)$.

Equation (A.4) is now transformed into

$$
\left[-\left(\frac{d}{d \xi_{j}}\right)^{2}+\left(p_{j}\left(\xi_{j}\right)+q_{j}\left(\xi_{j}\right)\right)\right] y_{j}\left(\xi_{j}\right)=y_{j}\left(\xi_{j}\right) .
$$

It is proved in [2, II] that $p_{j}\left(\xi_{j}\right)$ consists of a sum of terms having the form (A.8) $f(t, z)=c(1+\alpha(t, z))\left(z-V_{L}(t)\right)^{-\mu}\left(V_{L}^{\left(i_{1}\right)}(t)\right)^{j_{1}} \cdots\left(V_{L}^{\left(i_{m}\right)}(t)\right)^{j_{m}}$, where $c$ is a real constant,

$$
\mu \geqslant j+\sum_{l=1}^{m} j_{l}, \quad \sum_{l=1}^{m} i_{l} j_{l} \geqslant 2 j
$$


and $1+\alpha(t, z)$ is a product of factors of the form $\left(1-p_{l}\left(\xi_{l}\right)\right)^{-r}, 1 \leqslant l \leqslant j-1$, $r>0$. Hence (A.8), (A.9) and assumption (VL1) imply that

$$
\left|p_{j}\left(\xi_{j}\right)\right|=O\left(\left|z-V_{L}(t)\right|^{-j} t^{-\delta \Sigma_{l=1}^{m} i_{l} j_{l}}\right),
$$

uniformly in $z \in \Omega(I), t_{0} \leqslant t<\infty$. In particular, for $j=k$

$$
\begin{gathered}
\left|p_{k}\left(\xi_{k}\right)\right|=O\left(\left|z-V_{L}(t)\right|^{-k} t^{-2 k \delta}\right), \\
\left|q_{k}\left(\xi_{k}\right)\right|\left|\frac{d \xi_{k}}{d t}\right| \leqslant g(t) \leqslant C\left|V_{S}(t)\right| \cdot\left(1+\left|V_{L}(t)\right|\right)^{-1 / 2},
\end{gathered}
$$

where $g(t) \in L^{1}\left(t_{0}, \infty\right)$. Note that by (A.2), (A.5)

$$
d \xi_{k}=\left(z-V_{L}(t)\right)^{1 / 2} \prod_{l=1}^{k-1}\left(1-p_{l}\left(\xi_{l}\right)\right)^{1 / 2} d t,
$$

Finally, one can show that the curve $\Gamma_{k}$ is monotone increasing, namely

$$
\operatorname{Im}\left(\xi_{k}\left(t_{1}, z\right)-\xi_{k}\left(t_{2}, z\right)\right) \geqslant 0, \quad t_{1} \geqslant t_{2} .
$$

Equation (A.7) with $j=k$ is now written as a $2 \times 2$ first order system

$$
\begin{gathered}
w^{\prime}=\left(\begin{array}{cc}
0 & 1 \\
-1 & b\left(\xi_{k}\right)
\end{array}\right) w, \quad w=\left(\begin{array}{l}
y_{k}\left(\xi_{k}\right) \\
y_{k}^{\prime}\left(\xi_{k}\right)
\end{array}\right), \\
b\left(\xi_{k}\right)=p_{k}\left(\xi_{k}\right)+q_{k}\left(\xi_{k}\right),
\end{gathered}
$$

or, in a diagonalized form

$$
v^{\prime}=\left(\left(\begin{array}{cc}
i & 0 \\
0 & -i
\end{array}\right)+R\left(\xi_{k}\right)\right) v=\left(D+R\left(\xi_{k}\right)\right) v
$$

where

$$
\left|R\left(\xi_{k}\right)\right| \leqslant C\left(\left|p_{k}\left(\xi_{k}\right)\right|+\left|q_{k}\left(\xi_{k}\right)\right|\right) .
$$

Let $B$ be the Banach space of vector functions $f(t, z)$, continuous on $\left[t_{0}, \infty\right) \times$ $\Omega(I)$ and normed by

$$
\|f(t, z)\|=\sup _{\substack{z \in \Omega(I) \\ t_{0} \leqslant t<\infty}}\left|\exp \left(-i \xi_{k}(t, z)\right) f(t, z)\right|,
$$

and let $T$ be the transformation defined by

$$
(T f)(t, z)=\int_{t}^{\infty} \exp \left[\left(\xi_{k}(t, z)-\xi_{k}(s, z)\right) D\right] R\left(\xi_{k}\right) f(s, z) \frac{d \xi_{k}}{d s} d s
$$

It follows easily from the estimates (A.15), (A.11) and inequality (A.13) that $I+T$ is invertible if $t_{0}$ is sufficiently large. Furthermore, if $v$ solves

$$
v=\exp \left(i \xi_{k}(t, z)\right)\left(\begin{array}{l}
1 \\
0
\end{array}\right)-T v
$$

then it is a solution to (A.14) and tracing it back via $w$ and the functions $y_{j}\left(\xi_{j}\right)$ we obtain a solution of (A.1). It is an easy matter to check that this solution satisfies all the assertions of Lemma 3.1. In particular, note that by (A.17) and the estimates (A.15), (A11), if $f \in B, \exp \left(-i \xi_{k}(t, z)\right) T f$ satisfies uniformly an estimate of the type 
(3.2). Thus, by (A.18), the exponential term in the expansion (3.3) is justified if we put (see (A.12))

$$
1+a(t, z)=\prod_{l=1}^{k-1}\left(1-p_{l}\left(\xi_{l}\right)\right)^{1 / 2}
$$

$a(t, z)$ depends only on $V_{L}$, since only $V_{L}$ was involved in the construction of $p_{l}\left(\xi_{l}\right)$. (b) and (c) of the lemma follow immediately from (A.10), (A.11). (d) follows from the observation that $a(t, \lambda)$ involves $\lambda$ only through negative powers of $\left(\lambda-V_{L}(t)\right)$. Finally, note that definition (A.19) of $a(t, z)$ fits the modification factor involved in going from $v$ back to $y$ (see (A.6), (A.3)).

Proof of Lemma 3.2. The proof utilizes the same method as the previous proof. We point out briefly some differences. First, by transforming $t \rightarrow-t$ we may assume that we solve again over $\left[t_{0}, \infty\right)$. Instead of (A.2), (A.3), we define

$$
\xi_{1}(t, z)=\int_{t_{0}}^{t}\left(V_{L}(s)-z\right)^{1 / 2} d s, \quad y_{1}\left(\xi_{1}\right)=\left(V_{L}(t)-z\right)^{+1 / 4} y(t) .
$$

Instead of equation (A.4) we obtain now

$$
\left[\left(\frac{d}{d \xi_{1}}\right)^{2}+\left(p_{1}\left(\xi_{1}\right)+q_{1}\left(\xi_{1}\right)\right)\right] y_{1}\left(\xi_{1}\right)=y_{1}\left(\xi_{1}\right),
$$

where $p_{1}, q_{1}$ are the same as before except that $z-V_{L}$ is replaced by $V_{L}-z$.

We now follow exactly the same process of reduction, where again in (A.12) $\left(z-V_{L}\right)$ is replaced by $\left(V_{L}-z\right)$ and in (A.13) we take the real part instead of the imaginary part. We thus obtain equation (A.14) with thematrix $D=\left(\begin{array}{ll}1 & 0 \\ 0 & -1\end{array}\right)$. The norm (A.16) is now defined using the weight function $\exp \left(+\xi_{k}(t, z)\right)$ and a similar change in (A.18). The assertions of the lemma are now easily verified.

REMARK 1. Observe that if we modify the norm (A.16) by taking the weight function $\exp \left(-\xi_{k}(t, z)\right)$ (i.e., exponentially decaying), the resulting solution blows up exponentially (in fact, like $\exp \left(\xi_{k}(t)\right)$. Thus, the method can be applied to the unbounded solutions as well.

REMARK 2. For the case that $V_{L}$ is a polynomial our decaying solutions coincide with the "subdominant solutions" of [15, Chapter 2]. Note that assumption (VL3) has not been used in the derivation of the asymptotic expansion, which therefore applies to a polynomial of any order. In particular, for $V(t)=t^{2}$ we obtain the correct rate of decay of eigenfunctions of the harmonic oscillator (i.e., $\exp \left(-\frac{1}{2} t^{2}\right)$ ).

Proof of Lemma 3.3. To prove part (a), we modify the definition on the Banach space $B$ from the proof of Lemma 3.1: Let $B$ now be the space of vector functions $f(t, z)$, defined and continuous on $\left[t_{0}, \infty\right) \times I$ ( $z$ real) and so that $f$ is $m$-times continuously differentiable in $z$. The norm in $B$ (instead of (A.16)) is

$$
\|f(t, z)\|=\sup _{\substack{z \in I \\ t_{0} \leqslant t<\infty}} \sum_{j=0}^{m}\left|w(t)^{-j}\left(\frac{d}{d z}\right)^{j} f(t, z)\right|,
$$

where $w$ is given by (3.5). By Lemma 3.1(d) and (A.12) we have

$$
\left|\frac{d \xi_{k}}{d z}\right| \leqslant C w(t)
$$


Hence, if $f \in B$ and $0 \leqslant j \leqslant m$

$$
\left|\left(\frac{d}{d z}\right)^{j}\left(\exp \left(i \xi_{k}(t, z)\right) f(t, z)\right)\right| \leqslant C w(t)^{j}\|f\| .
$$

This inequality implies that under condition (3.6) the transformation $T$ defined by (A.17) is bounded in $B$ and if $t_{0}$ is sufficiently large $\|T\|<\frac{1}{2}$. Thus $I+T$ is invertible and equation (A.18) possesses a unique solution $v \in B$. Obviously, $v$ coincides pointwise with the $y^{+}(t, z)$ solution. Furthermore, equation (A.18) can be differentiated $m$-times with respect to $z$ and taking the above inequalities into consideration the expansion (3.7)-(3.8) follows easily.

REMARK 3. If $\lim _{t \rightarrow+\infty} V_{L}(t)=0$ the function $w(t)$ can be replaced by $t$. Our method is then identical with the one employed by Devinatz [5] in the study of oscillating potentials.

We now prove part (b) of the lemma by inspecting the proof of Lemma 3.2. We note that inequality (A.13), which is crucial in the proof of Lemma 3.1 (i.e., the estimate of $\|T\|$ in (A.17)), holds true if and only if $\operatorname{Im} z \geqslant 0$, namely $z \in \Omega(I)$. On the other hand, the inequality

$$
\operatorname{Re}\left(\xi_{k}\left(t_{1}, z\right)-\xi_{k}\left(t_{2}, z\right)\right) \geqslant 0, \quad t_{1} \geqslant t_{2},
$$

which replaces (A.13) in the proof of Lemma 3.2, holds true as long as $\operatorname{Re} z<V_{L}(t)$. Thus, we can repeat the proof of Lemma 3.2 with $\Omega(I)$ extended to $\Omega(I) \cup \overline{\Omega(I)}$ (where - denotes complex conjugation). Also we modify $B$ by the additional requirement that $f(t, z)$ is analytic in $z$ in a compact set containing $I$. Again, $T$ as defined by (A.17) is bounded in $B$ and the equation replacing (A.18) can be solved uniquely, yielding $y^{-}(t, z)$ as an analytic function in $z$ in a neighborhood of $I$. Since $I \subseteq \Lambda$ is compact we may assume that there exists a $\gamma>0$ such that $\operatorname{Re}\left(\xi_{k}(t, z)\right) \geqslant \gamma$ for $z$ in that neighborhood. The expansion (3.4) along with the assumption (3.9) imply now the exponential decay of $y^{-}(t, z)$. Inequality (3.10) follows of course by applying Cauchy's theorem to the analytic function $y^{-}(t, z)$.

\section{REFERENCES}

1. J. E. Avron and I. W. Herbst, Spectral and scattering theory of Schrödinger operators related to the Stark effect, Comm. Math. Phys. 52 (1977), 239-254.

2. M. Ben-Artzi, On the absolute continuity of Schrödinger operators with spherically symmetric, long-range potentials. I, II, J. Differential Equations 38 (1980), 41-60.

3. Unitary equivalence and scattering theory for Stark-like Hamiltonians (preprint).

4. M. Ben-Artzi and A. Devinatz, Spectral and scattering theory for the adiabatic oscillator and related potentials, J. Math. Phys. 20 (1979), 594-607.

5. A. Devinatz, The existence of wave-operators for oscillating potentials, J. Math. Phys. 21 (1980), 2406-2411.

6. J. D. Dollard and C. N. Friedman, Existence of the Moller wave-operators for $V(r)=\lambda r^{-\beta} \sin \left(\mu r^{\alpha}\right)$, Ann. Physics 111 (1978), 251-266.

7. N. Dunford and J. T. Schwartz, Linear operators. II, Wiley Interscience, New York, 1967.

8. T. A. Green and O. E. Lanford III, Rigorous derivation of phase shift formula for the Hilbert space scattering operator of a single particle, J. Math. Phys. 1 (1960), 139-148.

9. I. W. Herbst, Unitary equivalence of Stark Hamiltonians, Math. Z. 155 (1977), 55-70.

10. L. Hörmander, The existence of wave-operators in scattering theory Math. Z. 146 (1976), 69-91.

11. T. Kato, Perturbation theory for linear operators, Springer-Verlag, Berlin, Heidelberg and New York, 1966. 
12. M. Reed and B. Simon, Methods of modern mathematical physics. Vol. I: Functional analysis, Academic Press, New York and London, 1972.

13. __ Methods of modern mathematical physics. Vol. III: Scattering theory, Academic Press, New York and London, 1979.

14. P. A. Rejto and K. Sinha, Absolute continuity for a 1-dimensional model of the Stark Hamiltonian, Helv. Phys. Acta 49 (1976), 389-413.

15. Y. Sibuya, Global theory of a second order linear ordinary differential equation with a polynomial coefficient, North-Holland, New York, 1975.

16. B. Simon, Phase space analysis of simple scattering systems; extensions of some work of Enss, Duke Math. J. 46 (1979), 119-168.

17. K. Veselić and J. Weidmann, Potential scattering in a homogeneous electrostatic field, Math. Z. 156 (1977), 93-104.

18. K. Yajima, Spectral and scattering theory for Schrödinger operators with Stark effect, J. Fac. Sci. Univ. Tokyo Sect. IA 26 (1979), 377-389.

Department of Mathematics, Technion-Israel Institute of Technology, Haifa 32000, Israel 